\title{
Increasing Environmental Safety of Urbanized Territories Applying Recovery Absorber in Reduction of Emissions of Gas Station
}

\author{
E. Sokolova*, S. Koshkarev, R. Magomedov, O. Klimenko \\ North-Caucasus Federal University, engineering Institute, Stavropol, Russia \\ ${ }^{*}$ Corresponding author. E-mail: asokolova0584@mail.ru
}

\begin{abstract}
The issue of this article is improving the ecological safety of gas filling stations (GFS) that are placed within urban areas and the boundary of the sanitary protection zone. It has been determined that the main share of GFS' emissions is produced by petroleum products, especially when reservoirs are filled fuel storage tanks. It was proposed the way for reduce emissions from fuel storage tanks during the filled operation. It could be reach by applying of an apparatus for the absorption of gasoline vapors from GFS' emissions into the atmosphere. There presented the results by proposed devices for cleaning emissions GFS' in this article. It was obtained the regressions of recovery effectiveness for the improving design devices by mathematical processing for fuel oil and kerosene as work sorbent liquids. There are suggesting some approaches for modeling and engineering calculation's ways of such absorbing recovery devices in this article. The research results were implemented for design of full scale industrial set up that was installed and successful tested in one aspiration schemes GFS situated in Stavropol region of Russia. It was showed the reliability of the proposed device for practical use in the structure of the city municipal economy.
\end{abstract}

Keywords: gas station, city complexes, oil products, absorber.

\section{INTRODUCTION}

The number of gas filling stations (GFS) and volumes of gasoline fuel sales are increasing from year to year in the Russia. Volumes of gasoline fuel that sales though GFS was grow from 480 in 2015 to around 515 million tons in 2016 [1]. The GFS' number are 530 in Volgograd area and 705 in Stavropol region at the end of 2016 accordingly statistic data [2]. The calculating results of average amount emissions of harmful substances exhausting into the atmospheric air from the only average GFS could obtain around 10 tons per year $[3,4]$. The average volumes of these emissions could amount around 150 tons per year from GFS that are situated in Stavropol and up to 5000 tons per year from GFS, for example, that are placed in Volgograd region $[3,4]$. It should be note that GFS are situated inside city's development often $[4,5]$. Toluene, xylene and ethylbenzene are most dangerous harmful substances that vapor containing in the gasoline GFS' emissions. Growing up emissions' gross annual average and maximum one time volumes exhausting into atmospheric air from GFS harmful substances and add an additional negative human-made contribution to environmental pollution. There were present results of the measurements' concentrations of vapor benzene in the air at the different control point's border in the area of GFS' location in $[5,6]$. It was showed that vapor benzene concentrations surpass the allowable values of these ingredients' concentration Cmpcin 2- 3 times around in average. It is noted, for example, in $[7,8]$, that this problem exists and excess concentrations pollutants occur. In addition, there is a dangerous effect of these ingredients' at human health [9] for residential communities. Health risk characterization of exposure to benzene in service stations and petroleum refineries has been carried out in previous studies using guideline values set by various agencies was shown in [10]. Thus, this problem is actually and is under the attention of the scientific community. It is necessary to solve this problem to decrease emissions GFS the atmosphere. A certain number of technical activities in environment well known ways decreasing volumes of harmful emissions of GFS into the atmosphere were described in literature, for example, $[3,4]$. In $[11,12]$ was present the results of experimental study of proposed wet 
cleaning device [13] for the aspiration schemes to reduce dust and gaseous emissions. It was proposed to use this liquid device as absorber for trapping gas. In [14] was revealed the experimental tests of evaluating results of some kind combined absorbing device for trapping gaseous ingredients of GFS' vapor emission of aspiration schemes.

One of the aims of presented research is study evaluating results for a new modification of the device for trapping GFS' vapor gaseous ingredients too to decrease harmful vapor volume of the emissions reliable and acceptable. It was made an attempt to provide a description of the vapor recovery in the absorbing device to get reliable calculating approach. Another aim of the work was to fulfill test of hydro - mechanical properties of the device and to present their.

The one-parametrical quasi diffusion model could use for description of multiphase mass exchanged processes in the various kinds of constructive shape of apparatus [15, 16]. Such model for applied for modeling of the continuous dynamic trapping of gases from emissions of GFS by capturing absorptive device

$$
\partial \frac{\partial}{\partial x}\left(\frac{D \partial c}{\partial x}\right)-\frac{\partial(w c)}{\partial x}=\frac{\partial c}{\partial \tau}
$$

where $D$ - the value of effective diffusion coefficient of volume mixing of the liquid in the direction of its motion $\mathrm{x}$, $\mathrm{m} 2 / \mathrm{s}$.

$c$ - the value of the relative concentration of benzene vapor (benzene, toluene) in liquid sorbent along the length of the apparatus (the reduced length) the in the direction of its motion $\mathrm{x}, \mathrm{kg} / \mathrm{kg}$.

The quasi kinetics parameter of absorption as we mean the change in the relative concentration content for most sorbent liquids can be approximated by linearly decreasing rateor parameter of absorption $(\mathrm{dc} / \mathrm{d} \tau)=-\mathrm{kc}$ from a practical point of view. It is proposed that this kinetics parameter of absorption tied with the $\mathrm{x}$ direction length of the apparatus (along the reduced its length for moving sorbent liquids). In this case allow obtain solutions in the definite form of functions. Boundary conditions to solution of the Cauchy task (problem) were proposed and formulized by Dankverts. It frequently applied to describe the chemicaltechnological processes in different reactors (devices) [15 -17]

$$
\begin{array}{cc|c}
=w c & 0 \\
& x=0 & x=0
\end{array} \mid x=L
$$

where $w$ - The value of linear velocity of the moving layer of sorbent liquids in the direction of its motion $x$ of the apparatus, $\mathrm{m} / \mathrm{s}$.

$$
c=\left(1-c_{0}\right)\left[\begin{array}{l}
\frac{e^{\lambda_{1} x}}{1-\frac{D \lambda_{1}}{w} e^{L\left(\lambda_{1}-\lambda_{2}\right)}\left(e^{L\left(\lambda_{1}-\lambda_{2}\right)}+\frac{w}{D \lambda_{2}}-1\right)}- \\
-\frac{e^{\lambda_{2} x}}{\lambda_{2}\left(\frac{e^{L\left(\lambda_{1}-\lambda_{2}\right)}}{\lambda_{1}}-\frac{D}{w}\left(e^{L\left(\lambda_{1}-\lambda_{2}\right) x}+\frac{w}{D \lambda_{2}}-1\right)\right.}
\end{array}\right]
$$

where $\lambda_{1,2}=(w / 2 D) \pm \sqrt{\left((w / 2 D)^{2}+(k / D)\right)}$.

There is also another macro kinetic approach to analyze continuous processes of interfacial mass and heat transfer. The basis of this approach is study of the density of distribution $\rho(\tau)$ of liquid-sorbent dwell time in the apparatus. In the theoretical analysis the influence function (4) was used, which corresponds to the diffusion propagation of an instantaneously supplied point perturbation in an infinite medium stationary

$$
\rho(\tau)=\frac{1}{2 \sqrt{\pi D \tau}}\left[\exp \left(-\frac{(x-l)^{2}}{4 D \tau}\right)\right]
$$

In this case, the value of the mean final mass concentration of the vapor of gasoline Cout in the liquid sorbent discharged from the apparatus can be determined from the following integral dependence

$$
\bar{c}=\frac{1}{2 \sqrt{\pi D \tau}} \int_{0}^{\infty} \frac{c(\tau)}{\sqrt{\tau}}\left[\exp \left(-\frac{\left(L_{a}-w \tau\right)^{2}}{4 D \tau}\right)\right] d \tau,
$$

where $-\mathrm{w}$ is an equivalent length motion' rate of absorbing liquid flow in apparatus, $\mathrm{m} / \mathrm{s}$. It is most reliable way for determining the average final mass concentration of recovery gaseous ingredients benzene vapor (benzene, toluene) of GFS' is the use of the distribution' density over the time of its dwell time in the real apparatus of finite length (characteristic size diameter of apparatus Da, or length of apparatus La) directly obtained in experiments with the tracer (tinted liquid).

$$
w c_{0} \quad-D \frac{d c}{d x} \quad, \frac{d c}{d x}=
$$

$$
\bar{c}=\int_{0}^{\infty} c(\tau) \rho(\tau) d \bar{\tau}
$$




\section{MATERIALS AND METHODS OF RESEARCH}

A plot of the real (industrial) absorbing device [13] with overall dimensions $300 \mathrm{~mm}$ in diameter and 2500 $\mathrm{mm}$ around is shown on the fig. 1. The contact chambers have a diameter of $80 \mathrm{~mm}$, a height of 800 $\mathrm{mm}$. The apparatus was based on the proposed design, manufactured in full-scale (M 1: 1) design, preserving the geometric dimensions, relationships and forms of the laboratory model.

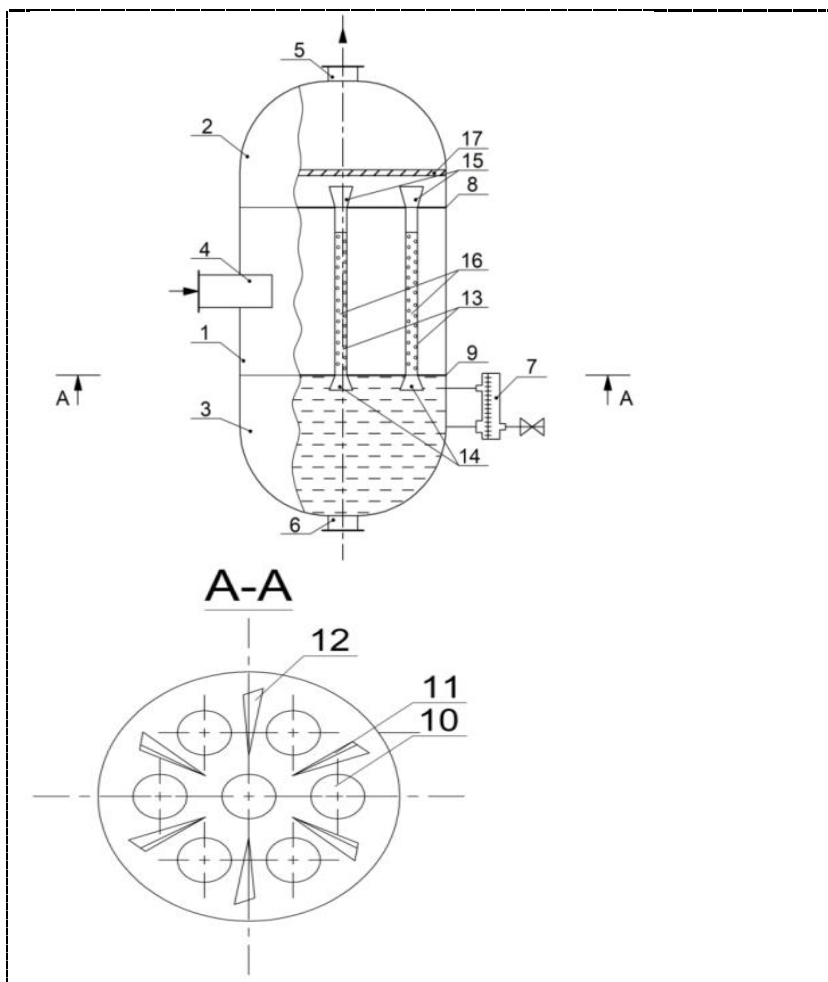

Figure 1 Scheme of proposed absorber for purification aspiration' emissions of gas station.

1 - the shell' hull; 2 - separation unit; 3 - pan with liquid absorber; 4 - inlet of polluted gas flow; 5 - outlet of clean gas flow; 6 - outlet of mud-draining waste liquid; 7 - liquid level controller in the sump; 8 and 9 horizontal baffle plate; 10 - fitting holes in the baffle plate for connection the contact chamber; 11 - vertex swirl blade at the bottom septum plate; 12 - slit slot; 13 contact chamber, 14 - confusers; 15 - diffusers; 16 ceramic nozzle (bulk loading) ; 17 - plate separator.

The scheme of this second kind of absorbing device [18] is shown in Figure 2.

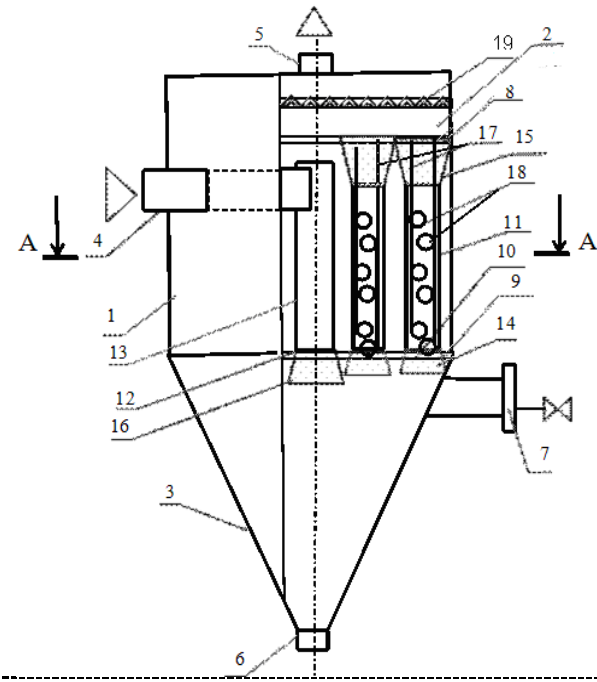

Figure 2 Plot scheme of wet cleaning device for separation dust particles from powder and gas flow reducing dust system of air emissions of aspiration scheme.

Characteristics and overall dimensions of the second kind of absorber were equal to first ones. Height (vertical length) of contact chamber of this second kind of absorbing device was the same (around $1000 \mathrm{~mm}$ ). The relationship diameter to height of contact chamber was the same in laboratory and real set up one's too. It was made to carry out scale modeling rules. Experiments were fulfilled on industrial set up at GFS' installation. Gas mixture of gasoline vapor (benzene, toluene and etc.) of GFS' and air that should clean was injected in inlet pipe of absorber in real time and nature field plant conditions. A fan fitted to outlet pipe in the upper part in separation block, pumped and made vacuum feeding gas mixture in the apparatus. Kerosene and fuel oil were treated as absorbing work liquids at the industrial set up GFS' installation. The height of the level of liquid sorbent in the pallet block and the level filling and lifting fluid in the contact chambers is maintained by feeding regulating device was controlled at scale one's unit visually. The height of with a liquid absorber was evaluated according to the scale of divisions applied to the walls of the chamber.

The aerodynamic resistance of the apparatus was determined from the difference in pressures measured with using the electronic kind of micro manometer' model "EPM LTA". Temperature, barometric pressure and relative air humidity were measured using a mercury expansion thermometer with a fission rate of $0.1^{\circ} \mathrm{C}$, an aneroid barometer and a psychrometer by standard techniques [19].The chromatograph "Color$500 \mathrm{M}$ " was used to determine the concentration of gasoline vapor in the gas-air mixture fed to the purification and after the apparatus [19]. The flow rate of the gas vapor -air mixture was regulated by means of an aspirating damper - choke. 
Effectiveness of the recovery of gas emissions in absorbing device could be defined by several factors. It were definite the most important determining and having opportunity to be real variable factors relatively recovery trapping of gas from gas flows need to be clean. It had been taken into investigation after analysis three factors for experimental evaluation procedure:

$\bar{\vartheta}_{c^{-}}$the relative velocity of the gasoline vapor and air mixture proceeded to be clean in the contact chamber, was related $1 \mathrm{~m} / \mathrm{s}$, correlated to initial bubbling regime equal varied from 1 to 6 ;

$\overline{\mathrm{h}}_{\mathrm{b}}$ - the relative height of the ceramic nozzle bulk loading in the contact chamber, referred to the height of the contact chamber, varied from 0.3 to 0.8 ;

$\overline{\mathrm{h}}_{\mathrm{lc}}$ - the liquid level of the lifting sorbent in the contact chamber, it was related to the height of the contact chamber and varying from 0 to 0.5 .

The determining factors $\mathrm{x} 1 \mathrm{x} 2$ and $\mathrm{x} 3$ could be describe by relations on recommendations [20]

$$
x_{1}=\frac{\bar{\vartheta}_{c}-\bar{\vartheta}_{c_{0}}}{\Delta \bar{\vartheta}}, x_{2}=\frac{\bar{h}_{b 3}-\bar{h}_{b_{0}}}{\Delta \overline{\mathrm{h}}_{b}}, x_{3}=\frac{\bar{h}_{l c}-\bar{h}_{l c_{0}}}{\Delta \overline{\mathrm{h}}_{l c}}
$$

The degree of gasoline vapor recovery (trapping effectiveness) and the hydro mechanical features of quasi-diffusion mixing characteristics of the liquidsorbent inside the proposed apparatus of the apparatus were considered and accepted as a response function.

The degree of capture was estimated as the ratio of the mass of trapped vapors to the mass of the vapors containing in the gas vapor-air mixture entering into absorber for the purification

$$
\eta=\frac{M_{1}-M_{2}}{M_{1}}
$$

where $M_{1}$, - mass of gasoline vapor in inlet $M_{2} \quad$ pipe before and in outlet branch pipes after apparatus, respectively, g.

Mass of gasoline vapor $M$ were determined

$$
M=c L
$$

where $c$ - gasoline vapor concentration, $\mathrm{mg} / \mathrm{m} 3$;

$L$ - flow consumption of vapor-air mixture, $\mathrm{m}^{3} / \mathrm{s}$,
The flow velocity $\vartheta_{c}$ in the contact chamber ducts was determined by the ratio

$$
\vartheta_{c}=\frac{L}{3600 N \frac{\pi D_{\mathrm{K}}^{2}}{4}}=\frac{L}{18,086 N} \cdot \mathrm{m} / \mathrm{s}
$$

where $D_{c} \quad$ - diameter of contact chamber, M;

$\mathrm{N} \quad-\quad$ number of contact chambers, pcs.

The flow velocity $\vartheta$ was measures in outlet branch pipe after apparatus to get value of flow consumption of vapor-air mixture $L$.

Macro kinetic approach to analyze continuous processes of interfacial mass transfer is based on evaluation of the hydro mechanical features of quasidiffusion mixing characteristics of the liquid-sorbent inside the proposed apparatus. The basis of this approach is study of the density of distribution $\rho(\tau)$ of liquid-sorbent dwell time in the apparatus. It need fulfill to use experimental data quasi-diffusion mixing coefficient determination in engineering calculations. Data of the longitudinal quasi-diffusion mixing coefficient of the liquid-sorbent $\mathrm{D}$ inside the proposed apparatus are obtained by marking method to "wash out" from the apparatus of the injected labeled (stained) tracer which was tinted liquid. A description of wellknown injected tracer's tinted liquid method and technique is available in the literature, for example, [16, 21]. The average residence dwell time tarof the liquidsorbent in the apparatus was determined experimentally at the facility $[16,21]$.

The mass amount in the material samples was determined by standard chromatographic methods [17]. The concentration of tracer (tinted liquid) in the isample $\rho(\mathrm{Ci})$ was the ratio of tracer mass (tinted liquid) $\mathrm{Mt}$ in the i-sample to the product of the total mass of the tracer (tinted liquid) in the input injected impulse M0 to the sampling for period of time for fixed interval of this sample $\Delta \tau \imath$

$$
\rho\left(C_{i}\right)=M_{t} /\left(M_{0} \Delta \tau_{i}\right)
$$

\section{RESULTS AND DISCUSSION}

The mathematical processing of the experimental data' results receiving at absorbing device [13] allow getting regression for the efficiency of gasoline vapor (benzene, toluene and etc. mixture) trapping and recovery:

- when using fuel oil as a work liquid sorbent for recovery gasoline vapor in GFS' emissions

$$
\begin{gathered}
\eta=0,8+0,028 \bar{v}_{c}\left(1-0,1 \bar{v}_{c}\right)+0,14 \bar{h}_{b}\left(2,16 \bar{h}_{b}-\right. \\
1)+0,148 \bar{h}_{l c}\left(\bar{h}_{l c}-0,15\right)
\end{gathered}
$$


- when using kerosene as a work absorbing liquid for recovery gasoline vapor GFS' emissions

$$
\begin{gathered}
\eta=0,798+0,028 \bar{v}_{c}\left(1-0,1 \bar{v}_{c}\right)+0,14 \bar{h}_{b}\left(2,16 \bar{h}_{b}-\right. \\
1)+0,148 \bar{h}_{l c}\left(\bar{h}_{l c}-0,15\right)
\end{gathered}
$$

There shown the experimental dependencies that characterize the degree' change of vapors-air mixture capture in the apparatus $\eta$ under various operating conditions of its operation within most the reliable and effective the device' work range in the case of using kerosene as a liquid sorbent at the fig. 3 and fig. 4 .

The significance and adequacy of the coefficients in presented regression's equations (12)- (13) were evaluated by the Fisher and Student criteria quantities by comparing the mathematically yielded values with standard tabular data of these numbers respectively [20].

In this paper we present the results obtained in a series of experiments at a laboratory facility [13] for using kerosene as an absorbing liquid for trapping gasoline vapor. Examples of the tracer "wash out" (run out) curves the density of distribution $\rho(\tau)$ as dependence for average residence dwell time $\tau$ arin the absorbing apparatus in set up [13] for low aero dynamics regimes are shown in Fig.5. Relative velocity of the gas gasoline vapor and air mixture proceeded to be cleaned in the contact chamber was equal around 2.
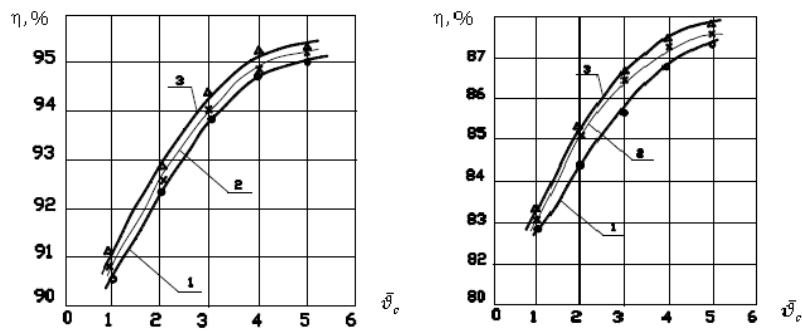

Figure 3 The degree of change in the efficiency of trapping and recovery of gasoline vapor ingredients $\eta$ from gas air mixture' flow velocity in the contact chamber in case applying kerosene as a liquid sorbent at $\bar{h}_{b}=0,8$, for: $1-\bar{h}_{l c}=0$; $2-\bar{h}_{l c}=0,25 ; 3-\bar{h}_{l c}=0,5$
Figure 4 The degree of change in the efficiency of trapping and recovery of gasoline vapor ingredients $\eta$ from gas air mixture' flow velocity in the contact chamber in case applying kerosene as a absorbing liquid at $\bar{h}_{b}=$ 0,5 , for: $1-\bar{h}_{l c}=0 ; 2$ $\bar{h}_{l c}=0,25 ; 3-\bar{h}_{l c}=0,5$

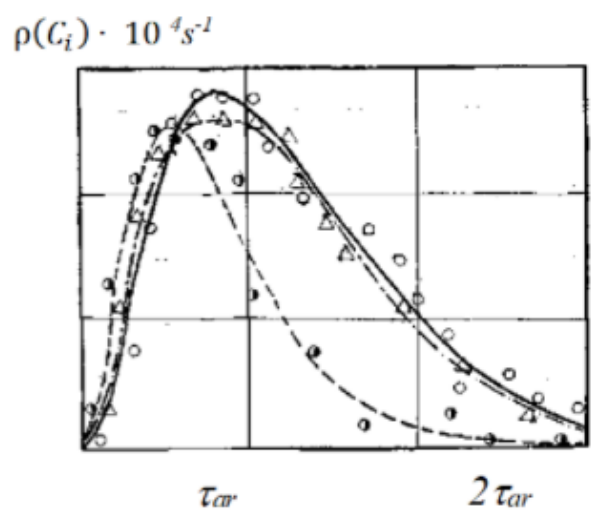

Figure 5 Changes in the of the density of distribution $\mathrm{r}(\mathrm{t})$ of the «wash out» tracer as a function of the average residence time tarfor use kerosene as a work absorbing liquid in the apparatus set up

Longitudinal mixing coefficient D was calculated from the criterion number Peclet characterizing the hydromechanical features of the motion of the absorbing liquid $\mathrm{Pe}=(\mathrm{w} \mathrm{La}) / \mathrm{Din}$ the apparatus, where $\mathrm{La}$ is the length of the apparatus [13]. The determination of the value number of the $\mathrm{Pe}$ criterion was made by calculating processing of the experimental data that was recommended $[16,21]$ due to relations

$$
\frac{1}{\mathrm{Pe}}=(1 \pm \sqrt{1+3 \sigma}) / 3
$$

where $\sigma^{2}=\alpha_{2}^{2}-\alpha_{1}^{2}, \alpha_{1}$ и $\alpha_{2}$ aremoments of the first and second order

$\alpha_{1}=\sum\left(\tau_{i} \rho\left(C_{i}\right)\right) / \rho\left(C_{i}\right), \quad \alpha_{2}=\sum\left(\tau_{i}^{2} \rho\left(C_{i}\right)\right) / \rho\left(C_{i}\right)$

The values of the coefficient of quasi diffusive mixing coefficient of tracer (tinted liquid) in liquidsorbent $D$ was varied depending on the aerohydrodymic regimes of relative velocity of the gas gasoline vapor and air mixture proceeded to be cleaned in the contact chamber and equivalent length motion' rate of absorbing liquid within apparatus $w(\mathrm{~m} / \mathrm{s})$.

\section{CONCLUSION}

1. It was founded that the maximal efficiency of the apparatus is reached when relative velocity of the gas gasoline vapor and air mixture proceeded to be cleaned within the contact chamber changed from $3.5-4.5 \mathrm{~m} / \mathrm{s}$. The maximal degree of recovery was obtained when height of bulk nozzle and a liquid sorbent is $50-80 \%$ of full dimension of the contact chambers.

2. Proposed apparatus and ways of its modeling and its engineering design could be applied in real practice with the reliable a solution of the problem of scaling with accepted effectiveness. Increasing productivity is reached by installation in devices necessary number of 
contact chambers with the fixed geometrical dimensions aerodynamic characteristics of the mode's flows (gases and liquid-sorbent should be within studied ranges). The results of the studies and was implemented in industrial aspiration scheme equipped proposed design device that showed effective the work for gasoline recovery trapping of vapors' GFS' emissions and reliable ones.

3. The values of the coefficient of quasi diffusive mixing coefficient of tracer (tinted liquid) in liquidsorbent $\mathrm{D}$ was varied depending on the aerohydrodymic regimes of relative velocity of the gas gasoline vapor and air mixture proceeded to be cleaned in the contact chamber and equivalent length motion' rate of absorbing liquid within apparatus. It was changed form $10-3$ to $10-2 \mathrm{~m} 2 / \mathrm{s}$.

4. The most reliable work was observed when kerosene use as a sorbent in the modification device [18], also average effectiveness was less. It is more preferable in practice use, because spherical nozzle isn't necessary to clean often, so that is easy operation with accepted effectiveness. Another reason to use kerosene as a sorbent is real possibility utilized waste kerosene as fuel, for example, in heat power generations stations.

\section{REFERENCES}

[1] Statistics kolichestvo2020dated2020galin asz, https://yandex.ru/search/?text=statistics\%20koliche stvo2020dated2020galin $\% 20$ asz \&lr=38\&clid=219 $2645 \&$ win $=330$ ).

[2] Number of filling stations (filling stations) in the constituent entity of the Russian Federation at the end of 2018, https://gks.ru/free_doc/new_site/business/transsv/azs.xls.

[3] S. Koshkarev, E. Sokolova, Experimental study of the hydrodynamic operating modes of the apparatus for capturing heavy hydrocarbons from emissions of filling stations, Alternative energy and ecology chapter, 11 (133) pp 71-74.

[4] S. Koshkarev, E. Sokolova, The efficiency's analysis of ways to decrease vapors of heavy hydrocarbons emissions out from gas filling station with use of results of modeling of their dispersion in the atmosphere, Alternative energy and ecology chapter, 11(133) pp. 52-55.

[5] S. Koshkarev, Reducing of emissions from gas filling stations based on the stratification modeling, Alternative energy and ecology chapter, 7, pp. 8292.

[6] V. Azarov, S. Koshkarev, E. Sokolova, To the justification of the size sanitary-a protective zone of petrol stations in reducing emissions of vapors of heavy hydrocarbons, Construction and reconstruction chapter, 4(48), pp. 57-59.

[7] F. Esmaelnejad, Monitoring of benzene, toluene, ethyl benzene, and xylene isomers emission from Shahreza gas stations in 2013, International Journal of Environmental Health Engineering chapter, (4)1, pp. 17.

M. Ebrahemzadih, Exposure Assessment to Benzene, Toluene, Ethyl Benzene and Xylene (BTEX) in Gas Stations in Central Region of Iran, Asian Journal of Water, Environment and Pollution chapter, 1(13), pp. 43-48.

[8] A. Rich, H. Orimoloye, Elevated atmospheric levels of benzene and benzene-related compounds from unconventional shale extraction and processing: human health concern for residential communities, Environmental health insights $\mathrm{C}$ EHIchapter, 10, pp. 33314.

[9] B. Edokpolo, Q. Yu, D. Connell, Health risk characterization for exposure to benzene in service stations and petroleum refineries environments using human adverse response data, Toxicology reports chapter, 2, pp. 917-927.

[10] V. Azarov, S. Koshkarev, Effectiveness' estimation of wet cleaner of dust decreasing emissions of expanded clay kiln oven, Magazine of Civil Engineering chapter, 2, pp. 18-32.

[11] S. Koshkarev, A. Evtushenko, S. Pushenko, Evaluation of solid particles slippage' amount throw out wet dust cleaning device in the dust removal system in building industry, ProcediaEng chapter, 165, pp. 1057-1069.

[12] S. Koshkarev, V. Azarov, S. Sokolova, Russia Patent 139, 122.

[13] S. Koshkarev, V. Azarov, I. Stefanenko, E. Sokolova, Applying absorption in environmental mechanics' decreasingof aspiration emissions of gas station, Applied Mechanics and Materials, 875, pp. 145-148

[14] V. Frolov, Modelling of drying of dispersed materials (Chemistry: Leningrad).

[15] A. Koshkarev, Continuous drying of dispersed materials in the device tray and cylindrical shape S.-Petersburg technological institute: S.-Petersburg

[16] S. Koshkarev, Modeling of cleaning of dust emission' in fluidized bed building aspiration' collector MATEC Web of Conferences. "International Science Conference SPbWOSCE2016, 07020. 
[17] S. Koshkarev, V. Azarov, Patent2575887 2016.

[18] L. Mishina, Methods of chemical, physicomechanical and metrological control, (Leningrad: Nedra) 1998.

[19] N. Johnson, Statistics and experimental design in engineering and science. Methods of experimental design. (Mir, Moscow) 1981.

[20] Yu. Zhorov, Modeling of physico-chemical processes of oil refining and petrochemistry. Chemistry, Moscow, 1978, pp. 376. 